\title{
Combined Treatment Strategy and Outcome of High Risk Neuroblastoma: Experience of the Children's Cancer Hospital-Egypt
}

\author{
Emad Moussa ${ }^{1}$, Mohamed Fawzy ${ }^{2 *}$, Alaa Younis ${ }^{3}$, Maged El Shafei ${ }^{3}$, Mohamed Saad Zaghloul ${ }^{4}$, \\ Naglaa El Kinaai ${ }^{5}$, Amal Refaat ${ }^{6}$, Noha Atta $^{7}$, Alaa El Haddad ${ }^{2}$ \\ ${ }^{1}$ Department of Pediatric Oncology, Children's Cancer Hospital-Egypt, Cairo, and Clinical Oncology Department, Faculty of Medi- \\ cine, Menufeya University, Menufeya, Egypt; ${ }^{2}$ Department of Pediatric Oncology, National Cancer Institute, Cairo University, and \\ Children's Cancer Hospital-Egypt, Cairo, Egypt; ${ }^{3}$ Department of Surgical Oncology, National Cancer Institute, Cairo University, and \\ Children's Cancer Hospital-Egypt, Cairo, Egypt; ${ }^{4}$ Department of Radiotherapy, National Cancer Institute, Cairo University, and \\ Children's Cancer Hospital-Egypt, Cairo, Egypt; ${ }^{5}$ Department of Pathology, National Cancer Institute, Cairo University, and Chil- \\ dren's Cancer Hospital Egypt, Cairo, Egypt; ${ }^{6}$ Department of Radiodiagnosis, National Cancer Institute, Cairo University, and Chil- \\ dren's Cancer Hospital-Egypt, Cairo, Egypt; ${ }^{7}$ Department of Research, Children's Cancer Hospital-Egypt, Cairo, Egypt. \\ Email: *mohammed.fawzy@57357.com
}

Received September $13^{\text {th }}, 2013$; revised October $12^{\text {th }}, 2013$; accepted October $20^{\text {th }}, 2013$

Copyright (C) 2013 Emad Moussa et al. This is an open access article distributed under the Creative Commons Attribution License, which permits unrestricted use, distribution, and reproduction in any medium, provided the original work is properly cited.

\begin{abstract}
Background: Neuroblastoma (NB) is remarkable for its wide spectrum of clinical behavior and biological characteristics in relation to outcome. The use of aggressive therapy, including autologous hematopoietic stem cell transplantation (HSCT) and the addition of isoretionin (cis-Retinoic Acid/cis-RA), has increased survival rates of patients with advanced disease. Methods: Pediatric 271 newly diagnosed high risk NB patients were prospectively enrolled into the study. Patients received neoadjuvant chemotherapy of alternating cycles: [cyclophosphamide, doxorubicin, vincristine $(\mathrm{CAdO})]$ and [etoposide, carboplatin]. Intensification courses of "ICE" (ifosfamide, carboplatin, and etoposide) regimen were administered to patients with bone marrow (BM) residual infiltration. Whenever safely feasible, complete surgical resection or debulking of the primary tumor was attempted for patients achieving partial response. Eligible patients underwent HSCT, while radiation therapy to the primary and metastatic sites, as well as maintenance with cis-RA was given for 6 months. Results: The median age of our patients was 2.8 years with male to female ratio of 1.65:1. At 4 years, the overall and event free survivals were $33.7 \%$ and $23.3 \%$ for the entire group under study, with significantly higher rates $(42.7 \%$ and $35.6 \%$, respectively) for HSCT patients $(\mathrm{n}=94 ; \mathrm{p}<0.001)$. The outcome was also significantly correlated with response to induction therapy, pathological subtype, as well as other variables. Conclusion: Myeloablative therapy followed by stem cell rescue is regarded as the most important goal of high risk NB treatment to improve survival till present. Each of consolidation HSCT, post induction disease status, as well as international neuroblastoma pathology classification (INPC) subtype was an independent predictive variable of survival. A collaborative effort with an emphasis on biologic characteristics of aggressive disease and tailored therapy needs to be strengthened to further our understanding of this disease.
\end{abstract}

Keywords: High-Risk Neuroblastoma; Treatment; Outcome

\section{Introduction}

Neuroblastoma (NB) accounts for more than $8 \%$ of malignancies in patients younger than 15 years of age, and is responsible for $15 \%$ of all pediatric oncology deaths [1]. The disease is remarkable for its wide spectrum of clinical behavior. Although substantial improve-

"Corresponding author. ment in outcome occurred in lower risk categories during the past few decades, the outcome for children with a high-risk (HR) disease has been improved only modestly having less than $40 \%$ long-term survival rates [2]. It is generally believed that biological characteristics are more relevant to the outcome in advanced NB than the type of chemotherapy or extent of resection [3]. However, the use of aggressive chemotherapy increased the survi- 
val rates of patients with advanced NB [4]. Myeloablative therapy and autologous hematopoietic stem cell rescue can significantly result in a better 5-year Overall Survival (OS) and Event Free Survival (EFS) than nonmyeloablative chemotherapy. While adding isoretionin (cis-Retinoic Acid/cis-RA) to consolidation therapy independently resulted in a significantly improved OS; analysis of consecutive trials from a single center demonstrated that combining cis-RA with monoclonal antibody (MoAb 3F8), and Granulocyte-Macrophage Colony Stimulating Factor (GM-CSF) has also improved survival significantly in HR-NB $[5,6]$. Chemotherapy dose-escalation strategy using tandem autologous Hematopoietic Stem Cell Transplantation (HSCT) was also encouraging for survival [7]. Other new chemotherapeutic agents such as irinotecan and paclitaxel are also evaluated in the treatment of advanced and refractory NB by ongoing clinical trials, however, further studies incorporating newer modalities are still required as well to reduce late adverse effects without jeopardizing survival outcome [8].

The purpose of this study was to evaluate the outcome of HR-NB in Egyptian children treated with aggressive multimodality approach that included HSCT and cis-RA.

\section{Patients and Methods}

\subsection{Patients}

Newly diagnosed and treated pediatric NB patients at The Children's Cancer Hospital-Egypt (CCHE/57357) were prospectively enrolled onto study from July 2007 till December 2011. The international criteria were used for risk stratification (International Neuroblastoma Staging System/INSS), and assessment of disease response defined as; complete response (CR), very good partial response (VGPR), partial response (PR), no response (NR), progressive disease (PD) or mixed response (MR); according to the International Neuroblastoma Response Criteria (INRC) [9]. Data was timely updated and analyzed at different checkpoints throughout study period.

\subsection{Workup}

- Tissue samples were processed and routinely stained with hematoxylin and eosin stain. Tissues were diagnosed as neuroblastoma and were classified histologically as poorly differentiated, undifferentiated or differentiating while ganglioneuroblastoma (GNB) were classified as nodular or intermixed. Tumor was classified according to the international neuroblastoma pathology classification (INPC/modified Shimada) into favorable or unfavorable; based on histology, mitosis/karyorrhexis index, and age [10]. Immunostains using BenchMark XT Ventana automated slide staining system were used either for confirmation; synaptophysin (most consistent in our lab), chromogranin, neuron specific enolase (NSE), CD56 or for differential diagnosis especially in undifferentiated and/or extra adrenal location (WT1, CD99, myogenin, LCA, MPO).

- Tissue samples were further studied for NMYC gene amplification in most of our patients. According to tissue feasibility, NMYC gene status was assessed by fluorescence in situ hybridization (FISH) on paraffin embedded tissue sections using Vysis LSI NMYC spectrum orange probe $2 \mathrm{p} 24.1$ (Abott molecular) according to manufacturer instructions [11]. Cases were categorized as having normal diploid pattern or amplified pattern in the form of homogenous staining region or double minutes (more than 10 copies).

- Other laboratory work included; serum ferritin, LDH, NSE, and urinary valinylmandelic acid (VMA) as baseline, prior to chemotherapy cycles, surgery, HSCT, and during follow up.

- Computed tomography (CT) was routinely done for tumor assessment at different checkpoints, while magnetic resonance imaging (MRI) was the preferred study for paraspinal and intracranial lesions.

- ${ }^{99} \mathrm{Tcm}$ diphosphonate bone scan and Metaiodobenzylguanidine (MIBG) scintigraphy were performed at presentation and by the end of induction therapy for evaluation of; bone, BM, and soft tissue disease.

- Bone marrow disease was assessed in all patients via bilateral bone marrow aspirates and biopsies.

\subsection{Inclusion Criteria}

- No prior systemic therapy except for localized emergency radiation to sites of life threatening or function-threatening disease and/or no more than one cycle of chemotherapy.

- Age: patients must be $\leq 18$ years of age at initial diagnosis.

- Established unequivocal diagnosis of NB or GNB morphology verified by histology and/or demonstration of clumps of tumor cells in BM with elevated urinary VMA.

- Presence of high risk defined features [12]:

- INSS Stage $2 \mathrm{a} / 2 \mathrm{~b}$ patients $\geq 12$ months of age with NMYC amplification, and unfavorable pathology.

- INSS Stage 3 patients $\geq 12$ months of age with NMYC amplification or unfavorable pathology.

- INSS Stage 4 with any of the following: a) Age $\geq$ 18 months regardless of biologic features, or, b) Age 12 - 18 months with any unfavorable or indeterminant/unsatisfactory/unknown biologic features.

- INSS Stage 3, 4, 4S patients $<12$ months of age with NMYC amplification.

- Patients $\geq 12$ months of age; initially diagnosed 
with INSS stage 1, 2, 4S who progressed to a stage 4 without interval chemotherapy.

- Adequate liver function: bilirubin $\leq 1.5 \mathrm{mg} / \mathrm{dL}$ and $\mathrm{ALT} \leq 300$.

- Adequate renal function: 24 hours urine collection for creatinine clearance $\geq 60 \mathrm{~mL} / \mathrm{min} / 1.73 \mathrm{~m}^{2}$ and a serum creatinine $\leq 1.5 \mathrm{mg} / \mathrm{dl}$.

- Normal cardiac function: ejection fraction $\geq 55 \%$ and fractional shortening $\geq 28 \%$ documented by echocargram.

- Informed consent: the patient's legally authorized guardian must acknowledge in writing that consent to receive chemotherapy, radiotherapy and surgery has been obtained, in accordance with local policies for $\mathrm{CCHE}$.

\subsection{Off Protocol Criteria}

- Progressive disease/no response on protocol therapy.

- Treatment limiting organ dysfunction.

\subsection{Treatment Plan}

Patients were started on upfront induction chemotherapy with "cyclophosphamide, doxorubicin, vincristine (CAdO)" alternating with "carboplatin and etoposide" for 6 cycles [13]. Patients failed to have their BM cleared of metastatic disease by end of upfront regimen or had less than PR after 4 cycles, were upgraded to receive additional 2 4 intensification courses of "ICE" [ifosfamide: 1800 $\mathrm{mg} / \mathrm{m}^{2} / \mathrm{d}\left(\mathrm{d} 1\right.$ - d5), carboplatin: $560 \mathrm{mg} / \mathrm{m}^{2}(\mathrm{~d} 1)$, and etoposide: $100 \mathrm{mg} / \mathrm{m}^{2} / \mathrm{d}(\mathrm{d} 1$ - $\left.\mathrm{d} 5)\right]$; guided with response. When safely feasible, surgical resection or debulking of the primary tumor was attempted between 5th and 6th chemotherapy cycles for patients achieved PR. By end of induction, consolidation with autologous HSCT was offered to eligible patients with at least PR and had their BM cleared of disease unless denied by parents. Radiation therapy was consistently delivered to the primary as well as postinduction MIBG avid metastatic bony sites. Maintenance cis-RA was given for 14 days q monthly at $160 \mathrm{mg} / \mathrm{m}^{2} /$ day for 6 months following HSCT or post induction for non transplant patients attained CR/VGPR/PR [14].

\subsection{Statistical Analysis}

All data were analyzed using SPSS 16.0 software (SPSS, Chicago, IL, USA). Correlations were analyzed using $\chi^{2}$ test. OS was calculated from date of diagnosis to the date of death for any cause, and EFS was defined as the time from diagnosis to the time of first occurrence of relapse, progression, or death.

The Kaplan-Meier curves were plotted to calculate 5-year survival curves, and log-rank test was used to estimate the differences. Factors known to be associated with prognosis were tested in univariate analysis. Varia- bles that were found to be significant in univariate analysis were then entered in a multivariate Cox proportional hazards regression model to identify those with independent prognostic information for EFS and OS. The " $P$ " value was defined as statistically significant if $<0.05$.

\section{Results}

Two hundreds and seventy one patients were enrolled during our study period. Patients age ranged between 2 months and 12.7 years (Median: 2.8 years) at time of diagnosis; 169 males (62.4\%) and 102 females $(37.6 \%)$ with a ratio of $1.65: 1$.

Histologically, NB was confirmed in 251 patients (92.6\%); 200 cases were unfavorable according to INPC with poorly differentiated morphology, while GNB was seen in 14 patients $(5.2 \%)$. Diagnosis was established in another 6 patients $(2.2 \%)$ by infiltrated BM biopsy and elevated urinary VMA. As regards NMYC gene status, 82 of studied cases showed an amplified gene (Figure 1); 2/82 showed mosaic pattern.

Abdominal tumor was the most common primary site of disease found in 245 of the study patients (90.4\%), with adrenal origin seen in 190 patients $(70.1 \%)$ while 55 $(20.3 \%)$ had extra-adrenal locations. BM was the most common site of metastasis which seen in $53.5 \%$ of patients. Clinicopathological data are presented in Table 1.

Local surgical control (complete/partial) was feasible in 157 patients. Due to early death or lost follow up, 23 patients were not evaluable for induction response whereas disease status for $248 / 271$ evaluable patients showed: 4 CR (1.6\%), 14 VGPR (5.6\%), 177 PR (71.4\%), 25 NR (10\%), 28 PD (11.3\%). Collectively, objective response (OR) was 78.6\% (CR + VGPR + PR). Five of the 271 study patients died before receiving any treatment and thus were excluded from survival analysis. The 4 years OS for all valid patients was $33.7 \%$ (median survival: 31.1 months; 95\% CI: 24.3 - 37.9), while EFS was

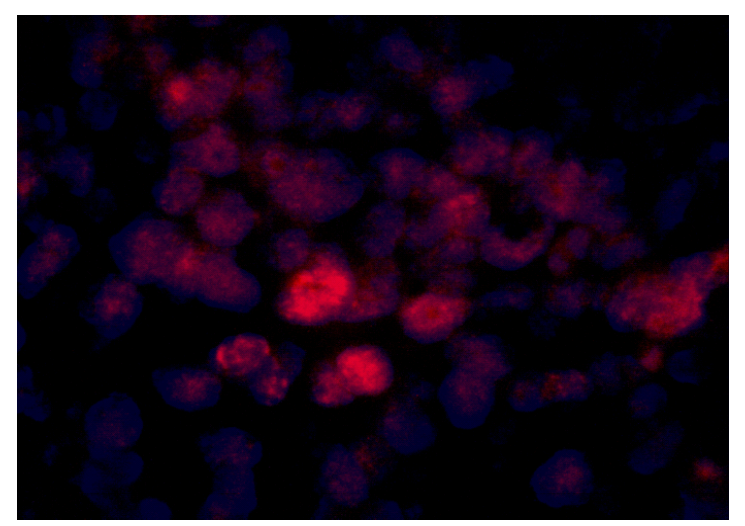

Figure 1. Amplified NMYC gene by fluorescence in situ hybridization (FISH) shows formation of fluorescent signal clusters $(\times 1000)$. 
Table 1. Clinicopathological data of the 271 high risk neuroblastoma study patients.

\begin{tabular}{|c|c|c|}
\hline Variable & $\begin{array}{c}\text { Number } \\
\text { (Total } \mathbf{n}=271)\end{array}$ & $\begin{array}{c}\text { Percentage } \\
(\%)\end{array}$ \\
\hline \multicolumn{3}{|l|}{ Primary site } \\
\hline Abdominal & 245 & 90.4 \\
\hline Mediastinal & 18 & 6.6 \\
\hline Others & 8 & 3 \\
\hline \multicolumn{3}{|l|}{ Pathology } \\
\hline NB & 251 & 92.6 \\
\hline GNB & 14 & 5.2 \\
\hline Not applicable & 6 & 2.2 \\
\hline \multicolumn{3}{|l|}{ INPC } \\
\hline Favorable & 22 & 8.1 \\
\hline Unfavorable & 200 & 73.9 \\
\hline Not applicable & 49 & 18 \\
\hline \multicolumn{3}{|l|}{ Stage (INSS) } \\
\hline Stage 2 & 1 & 0.4 \\
\hline Stage 3 & 57 & 21 \\
\hline Stage 4 & 199 & 73.4 \\
\hline Stage 4S & 14 & 5.1 \\
\hline \multicolumn{3}{|l|}{ NMYC } \\
\hline Amplified & 82 & 30.3 \\
\hline Not-amplified & 113 & 41.7 \\
\hline Not-applicable & 76 & 28 \\
\hline \multicolumn{3}{|l|}{ Secondary sites } \\
\hline \multicolumn{3}{|l|}{ Bone } \\
\hline No & 227 & 83.8 \\
\hline Yes & 44 & 16.2 \\
\hline \multicolumn{3}{|l|}{ CNS } \\
\hline No & 256 & 94.5 \\
\hline Yes & 15 & 5.5 \\
\hline \multicolumn{3}{|l|}{ Distant LNs } \\
\hline No & 243 & 89.7 \\
\hline Yes & 28 & 10.3 \\
\hline \multicolumn{3}{|l|}{ BM } \\
\hline Positive & 145 & 53.5 \\
\hline Negative & 94 & 34.7 \\
\hline Not-applicable & 32 & 11.8 \\
\hline
\end{tabular}

INPC; International Neuroblastoma Classification, INSS; International Neuroblastoma Staging System, NB; neuroblastoma, GNB; ganglioneuroblastoma, NMYC; oncogene, CNS; central nervous system, LN; lymph node, $\mathrm{BM}$; bone marrow.

$23.3 \%$ at a median follow up of 18.59 months (range: 0.2 - 57.9 months).

As 195 patients showed OR by end of induction; 94 underwent HSCT and their outcome was compared to the non transplant group $(\mathrm{n}=101)$. Statistically significant higher OS (42.7\%) was reported for transplant patients (median survival: 42.5 months; 95\% CI: 34.1 - 50.9) and EFS (35.6\%), compared respectively to $27.6 \%$ (median survival: 20.5 months; 95\% CI: $12.5-28.5 ; P<0.001)$ and $15.5 \%(P<0.001)$ for the non transplant patients (Table 2).

Although statistically insignificant, both OS and EFS were at higher rates among patients received intensified induction with additional courses of "ICE" chemotherapy prior to HSCT $(n=28)$ compared to those who had no "ICE" before transplant (respectively, 57.2\% v 36.8\%; $P$ $=0.312$ and $47.9 \% \mathrm{v} 30.3 \% ; P=0.128$; median survival of $49 \mathrm{v} 40.3$ months).

Both OS and EFS were also correlated with response to induction therapy, extent of primary tumor resection, and INPC (Table 2). Comparing induction responders (OR) to non-responders (NR and PD) there was a much superior OS and EFS for patients with OR $(P=0.00$ and $P=0.001$, respectively). The outcome was also better in patients underwent surgical resection of their primary tumor compared to others who did not; the OS and EFS were respectively $40 \%$ and $20.9 \%$ in the former versus $26 \%$ and $9.1 \%$ in the latter $(P=0.00$, each $)$. As well, a highly significant difference in survival rates was found between favorable and unfavorable INPC patients (respectively, $81.6 \% \mathrm{v} 28.9 \% ; P=0.001$ and $65.5 \% \mathrm{v}$ $13.3 \% ; P=0.00)$. Amplification of NMYC gene compared to normal NMYC and NB histological subtype $(P$ $=0.00$ and $P=0.044$, respectively).

In multivariate analysis each of; consolidation HSCT, post induction disease status (response), and INPC based pathology subtype was an independent prognostic variable predicting survival (Figures 2-4).

On the other hand, EFS was significantly affected by stage, age, and CNS metastasis. Whereas EFS was $64.3 \%$ for stage $4 \mathrm{~S}$ disease, it was respectively $42.4 \%$ and $8.1 \%$ for stages 3 and $4(P=0.00 \%)$. Patients younger than 18 months had EFS of $34.1 \%$ compared to $11.1 \%$ for older age patients $\geq 18$ months $(p=0.047 \%)$.

Among secondary sites of metastasis, CNS was predictive of lower EFS in comparison to other sites $(P=$ $0.002)$. Although statistically non significant; nominally higher OS was reported with mediastinal tumors (46.8\%) compared to abdominal origin (34.1\%), and other sites $(25 \%),(P=0.141)$.

The abdominal extra-adrenal sites had higher OS than adrenal tumors (46.6\% vs. $30 \%$ respectively; $P=0.183)$, and only a borderline significance of improvement in OS as well as EFS was related to radiation therapy to the primary and metastatic sites (Table 2).

\section{Discussion}

Approximately, $40 \%$ of NB tumors are classified as HR using current risk stratification criteria that carry very poor outcomes despite the use of aggressive therapies [15]. Until recently, the best outcome reported for 
Table 2. Impact of epidemiologic and clinicopathological variables on overall and event free four years survival in high risk neuroblastoma study patients.

\begin{tabular}{|c|c|c|c|c|}
\hline Variable & Overall Survival & $P$-Value & Event Free Survival & $P$-Value \\
\hline Gender & & 0.81 & & 0.646 \\
\hline Female & $37 \%$ & & $20.8 \%$ & \\
\hline Male & $33.3 \%$ & & $15.2 \%$ & \\
\hline Age & & 0.954 & & $0.047^{*}$ \\
\hline$<18$ months & $45 \%$ & & $34.1 \%$ & \\
\hline$\geq 18$ months & $28.2 \%$ & & $11.1 \%$ & \\
\hline Primary site & & 0.141 & & 0.903 \\
\hline Abdominal & $34.1 \%$ & & $15.9 \%$ & \\
\hline Mediastinal & $46.8 \%$ & & $29.6 \%$ & \\
\hline Others & $25 \%$ & & $25 \%$ & \\
\hline Abdominal sites & & 0.141 & & 0.906 \\
\hline Adrenal & $30.2 \%$ & & $16 \%$ & \\
\hline Extra-adrenal & $50.2 \%$ & & $13.7 \%$ & \\
\hline Pathology & & $0.044^{*}$ & & 0.311 \\
\hline NB & $34.5 \%$ & & $18.3 \%$ & \\
\hline GNB & $42.2 \%$ & & $12.2 \%$ & \\
\hline INPC & & $0.001^{*}$ & & $0.00^{*}$ \\
\hline Favorable & $81.6 \%$ & & $65.5 \%$ & \\
\hline Unfavorable & $28.9 \%$ & & $13.3 \%$ & \\
\hline INSS Stage & & 0.062 & & $0.00^{*}$ \\
\hline Stage 3 & $53.2 \%$ & & $42.4 \%$ & \\
\hline Stage 4 & $27.4 \%$ & & $8.1 \%$ & \\
\hline Stage 4S & $55.6 \%$ & & $64.3 \%$ & \\
\hline NMYCA & & $0.00^{*}$ & & 0.64 \\
\hline Yes & $31 \%$ & & $22.9 \%$ & \\
\hline No & $36 \%$ & & $17.7 \%$ & \\
\hline Bone metastasis & & 0.107 & & 0.085 \\
\hline Yes & $14.7 \%$ & & $6.2 \%$ & \\
\hline No & $37.9 \%$ & & $19.5 \%$ & \\
\hline CNS metastasis & & 0.054 & & $0.002^{*}$ \\
\hline Yes & $39.4 \%$ & & $6.7 \%$ & \\
\hline No & $34.8 \%$ & & 17.8 & \\
\hline Distant lymph nodes & & 0.548 & & 0.317 \\
\hline Yes & $36 \%$ & & $10.2 \%$ & \\
\hline No & $36.9 \%$ & & $17.5 \%$ & \\
\hline Post-induction disease status (response) & & $0.00^{*}$ & & $0.001^{*}$ \\
\hline $\mathrm{OR}(\mathrm{CR}+\mathrm{VGPR}+\mathrm{PR})$ & $43 \%$ & & $18.4 \%$ & \\
\hline $\mathrm{NR}+\mathrm{PD}$ & $12 \%$ & & $11.2 \%$ & \\
\hline Surgery & & $0.00^{*}$ & & $0.00^{*}$ \\
\hline Yes (complete/partial) & $40 \%$ & & $20.9 \%$ & \\
\hline No & 26 & & $9.1 \%$ & \\
\hline Radiotherapy & & 0.05 & & 0.052 \\
\hline Yes & $36.3 \%$ & & $14.3 \%$ & \\
\hline No & $39.9 \%$ & & $27.5 \%$ & \\
\hline HSCT & & $<0.001^{*}$ & & $<0.001^{*}$ \\
\hline Done & $42.7 \%$ & & $35.6 \%$ & \\
\hline Not done & $26.8 \%$ & & $15.5 \%$ & \\
\hline
\end{tabular}

INPC; International Neuroblastoma Classification, INSS; International Neuroblastoma Staging System, NB; neuroblastoma, GNB; ganglioneuroblastoma, NMYCA; amplified NMYC, CNS; central nervous system, HSCT; hematopoietic stem cell transplantation, OR; objective response, NR; no response, PD; progressive disease, ${ }^{*}$ statistically significant. 


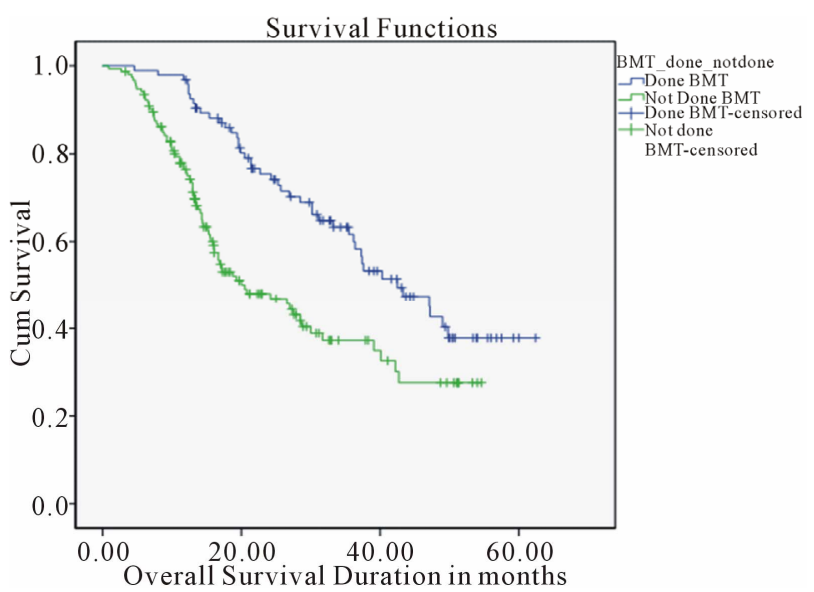

Figure 2. Overall survival for high risk neuroblastoma patients by transplant $(n=94)$ versus non-transplant $(n=177$; $P<0.001)$.

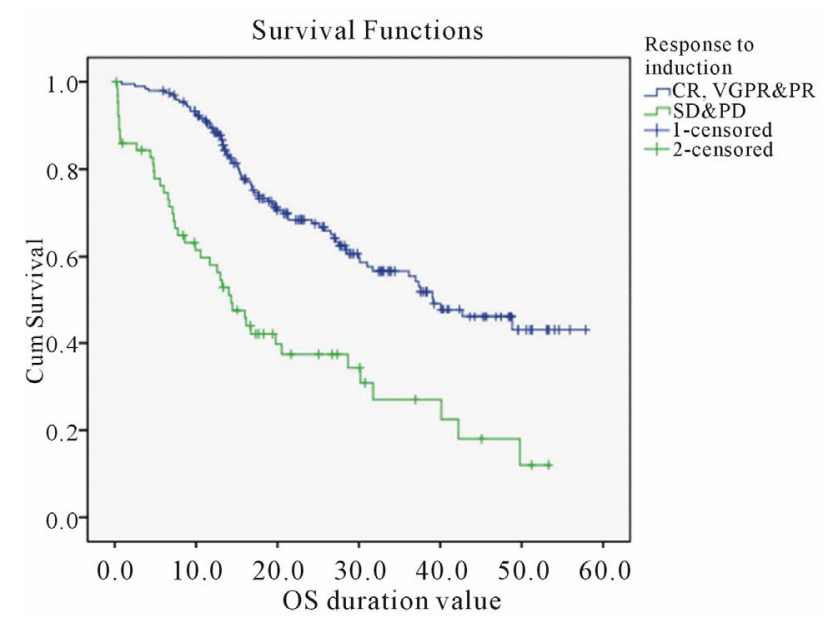

Figure 3. Overall survival for high risk neuroblastoma patients according to post induction response (complete response + very good partial response + partial response versus no response + progressive disease; $P=0.00$ ).

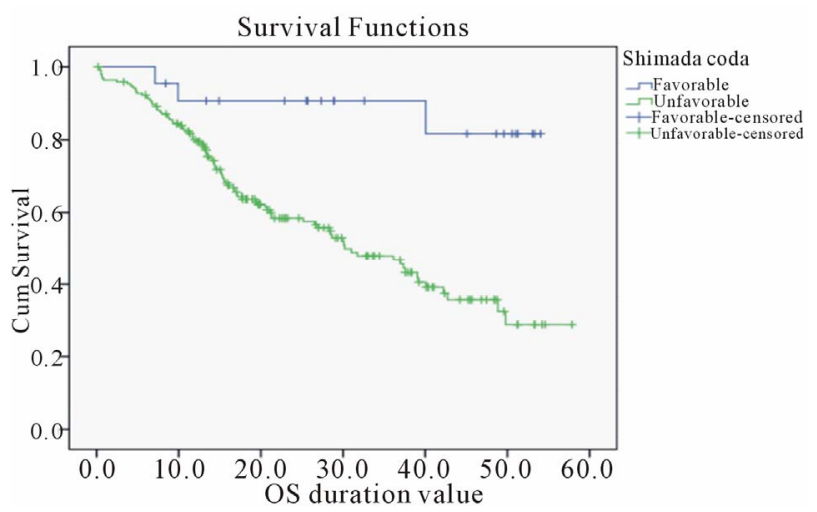

Figure 4. Overall survival for high risk neuroblastoma patients by pathology classification; favorable versus unfavorable $(P=0.001)$.
HR-NB was achieved with intensive combination induction chemotherapy and surgery; followed by myeloablative therapy, hematopoietic stem cell rescue, and cis-RA as a differentiating agent [16]. Our data showed that the 4 years OS for the entire HR-NB study patients was $33.7 \%$ and EFS of $23.3 \%$ including both transplant and non transplant patients. Similarly, an earlier Japanese NB study group reported a five-year OS outcome of 34.4\% for stage 4 disease patients [17], whereas a more recent study from Singapore reported a 5 years OS of only $28.2 \%$ for HR-NB [18].

Significant survival benefit found in a subgroup of our patients was ought to HSCT. The OS for patients underwent HSCT $(42.7 \%, \mathrm{n}=94)$ was significantly higher compared to those who did not $(27.6 \%, \mathrm{n}=177 ; \mathrm{P}<$ 0.001; Figure 2).

The EFS for transplanted patients $(35.6 \%)$ was also significantly higher than for other patients received no transplant $(15.5 \% ; P<0.001)$.

Consistently, in a well conducted prospective randomized clinical trial by Children's Oncology Group (COG), the EFS for patients randomly assigned to consolidation ABMT was significantly higher than for those randomly assigned to chemotherapy; the 5-year EFS was 30\% \pm $4 \%$ versus $19 \% \pm 3 \%$, respectively $(P=0.04)$.

Isoretinoin has independently resulted in a significantly improved OS when given following consolidation therapy. The 5-year OS of patients assigned to ABMT/ cis-RA was $59 \% \pm 8 \%$; versus $41 \% \pm 7 \%$ for $\mathrm{ABMT} /$ no cis-RA [5].

Chimeric anti-GD2 MAb (Ch14.18) tested in a large phase III COG randomized clinical trial in combination with IL-2 and GM-CSF; was associated with an improved 2 year EFS and OS in comparison to standard maintenance therapy $(66 \% \mathrm{v} 46 \%$ and $86 \%$ v $75 \%$, respecttively) [19].

As shown in Table 2, Post induction OR had a statistically significant positive impact when compared to NR and PD; on both OS (43\% v $12 \% ; P=0.00)$ and EFS $(18.4 \% \mathrm{~V} 11.2 \% ; P=0.001)$ that found to be in consistence with other investigators [20]. Our data also showed that OS and EFS were improved by resection of primary tumor compared to no or less than partial tumor resection; (40\% v $26 \%$ and $20.9 \%$ v $9.1 \%$, respectively; $P=0.00$; each). Though previous reports mentioned a controversial role for aggressive tumor resection [21,22], improved local control and OS rates in correlation to complete gross resection of the primary tumor were reported by La Quaglia et al., in stage 4 NB [23]. This was contradicted in a recent Finnish study which reported that OS in HR-NB patients was rather related to objective chemotherapy response, while no significant impact was found for complete excision of the primary tumor [24]. 
Unfavorable histological differentiation, amplified NMYC gene, CNS metastasis, and older age at diagnosis were all shown to have a significantly negative impact on the survival of our patients (Table 2).

While unfavorable INPC histology was associated with poorer OS and EFS than favorable subtype, CNS found to be the only metastatic site to show significant correlation to patients outcome as regards EFS $(P=$ $0.002)$. Similarly, EFS was significantly lower among patients $\geq 18$ months of age $(P=0.047)$ that was supported by other reports stated that age at diagnosis was one of the single most important indicators of survival in NB $[3,25]$. Unfavorable clinical variables such as; age above18 months and advanced stage were found to be closely associated with poor biologic risk factors including unfavorable histopathology, MYCN amplification, 1p and $11 \mathrm{q}$ loss of heterozygosity, as well as other partial chromosomal deletions [26].

\section{Conclusion}

In spite of aggressive therapy, HR-NB carried a discouraging survival outcome. The prognosis for high risk NB remained poor but myeloablative therapy followed by stem cell rescue is regarded as the most important goal of high risk NB treatment to improve survival till present. Each of consolidation HSCT, post induction disease status, as well as INPC-based pathological subtype was an independent predictive variable of survival. A collaborative effort with an emphasis on biologic characteristics of aggressive disease and tailored therapy needs to be strengthened to further our understanding of this disease.

\section{REFERENCES}

[1] S. L. Cohn, "Surveillance, Epidemiology, and End Results (SEER) Database," 2007, National Cancer Institute. http://seer.cancer.gov

[2] S. L. Cohn, A. D. J. Pearson, W. B. London, T. Monclair, P. F. Ambros, G. M. Brodeur, A. Faldum, B. Hero, T. Iehara, D. Machin, V. Mosseri, T. Simon, A. Garaventa, V. Castel and K. K. Matthay, "The International Neuroblastoma Risk Group (INRG) Classification System: An INRG Task force Report," Journal of Clinical Oncology, Vol. 27, No. 2, 2009, pp. 289-297. http://dx.doi.org/10.1200/JCO.2008.16.6785

[3] M. Kubota, M. Yagi, S. Kanada, N. Okuyama, Y. Kinoshita, S. Yamazaki, K. Asami, A. Ogawa and T. Watanabe, "Long-Term Follow-Up Status of Patients With Neuroblastoma after Undergoing Either Aggressive Surgery or Chemotherapy-A Single Institutional Study," Journal of Pediatric Surgery, Vol. 39, No. 9, 2004, pp. 1328-1332. http://dx.doi.org/10.1016/j.jpedsurg.2004.05.012

[4] M. Kaneko, Y. Tsuchida, H. Muqishima, N. Ohnuma, K. Yamamoto, K. Kawa, M. Iwafuchi, T. Sawada and S.
Suita, "Intensified Chemotherapy Increases the Survival Rates in Patients with Stage 4 Neuroblastoma with MYCN Amplification," Journal of Pediatric Hematology/Oncology, Vol. 24, No. 8, 2002, pp. 613-621. http://dx.doi.org/10.1097/00043426-200211000-00004

[5] K. K. Matthay, C. P. Reynolds, R. C. Seeger, H. Shimada, E. S. Adkins, D. Haas-Kogan, R. B. Gerbing, W. B. London and J. G. Villablanca, "Long-Term Results for Children with High-Risk Neuroblastoma Treated on a Randomized Trial of Myeloablative Therapy Followed by 13-cis-Retinoic Acid: A Children's Oncology Group Study," Journal of Clinical Oncology, Vol. 27, No. 7, 2009, pp. 1007-1013. http://dx.doi.org/10.1200/JCO.2007.13.8925

[6] B. Kushner, K. Kramer, S. Modak and N. K. Cheung, "Anti-GD2 Monoclonal Antibody 3F8 Plus Granulocyte Macrophage Colony Stimulating Factor for Primary Refractory Neuroblastoma in Bone Marrow," Proceedings of ASCO, Vol. 25, 2007, p. 9502.

[7] K. W. Sung, M. H. Son, S. H. Lee, K. H. Yoo, H. H. Koo, J. Y. Kim, E. J. Cho, S. K. Lee, Y. S. Choi, D. H. Lim, J. S. Kim and D. W. Kim, "Tandem High-Dose Chemotherapy and Autologous Stem Cell Transplantation in Patients with High-Risk Neuroblastoma: Results of SMC NB-2004 Study," Bone Marrow Transplant, Vol. 48, No. 1, 2013, pp. 68-73. http://dx.doi.org/10.1038/bmt.2012.86

[8] G. Surico, P. Muggeo, F. De Leonardis and N. Rigillo, "New Paclitaxel Cisplatin Based Chemotherapy Regimen for Advanced Stage, Recurrent, or Refractory Neuroblastoma-Preliminary Report," Medical and Pediatric Oncology, Vol. 40, No. 2, 2003, pp. 130-132. http://dx.doi.org/10.1002/mpo.10106

[9] G. M. Brodeur, J. Pritchard, F. Berthold, N. L. Carlsen, V. Castel, R. P. Castelberry, B. De Bernardi, A. E. Evans, M. Favrot, F. Hedborg, et al., "Revisions of the International Criteria for Neuroblastoma Diagnosis, Staging and Response to Treatment," Journal of Clinical Oncology, Vol. 11, 1993, pp. 1466-1477.

[10] O. Burques, S. Navarro, R. Noquera, A. Pellín, A. Ruiz, V. Castel and A. Llombart-Bosch, "Prognostic Value of the International Neuroblastoma Pathology Classification in Neuroblastoma (Schwannian Stroma-Poor) and Comparison with Other Prognostic Factors: A Study of 182 Cases from the Spanish Neuroblastoma Registry," Virchows Archives, Vol. 449, No. 4, 2006, pp. 410-420. http://dx.doi.org/10.1007/s00428-006-0253-y

[11] Y. Hachitanda, M. Saito, I. Mori and M. Hamazaki, “Application of Fluorescent in Situ Hybridization to Detect $\mathrm{N}$-myc Gene Amplification on Paraffin-Embedded Tissue Sections of Neuroblastoma," Medical and Pediatric Oncology, Vol. 29, 1997, pp. 135-138.

[12] G. M. Brodeur, M. D. Hogarty, Y. P. Mosse and J. M. Maris, "Neuroblastoma," In: P. A. Pizzo, P. C. Adamson and D. G. Poplack, Eds., Principles and Practice of Pediatric Oncology. 6th Edition, Lippincott Williams and Wilkins, Philadelphia, 2011, pp. 886-922.

[13] B. H. Kushner, R. J. O'Reilly, M. LaQuaglia and N. K. Cheung, "Dose-Intensive Use of Cyclophosphamide in Ablation of Neuroblastoma," Cancer, Vol. 66, 1990, pp. 1095-1100. 
http://dx.doi.org/10.1002/1097-0142(19900915)66:6<109 5::AID-CNCR2820660603>3.0.CO;2-0

[14] G. J. Veal, M. Cole, J. Errington, A. D. Pearson, A. B. Foot, G. Whyman and A. V. Boddy, "UKCCSG Pharmacology Working Group. Pharmacokinetics and Metabolism of 13-Cis-Retinoic Acid (Isotretinoin) in Children with High-Risk Neuroblastoma-A Study of the United Kingdom Children's Cancer Study Group," British Journal of Cancer, Vol. 96, No. 3, 2007, pp. 424-431. http://dx.doi.org/10.1038/sj.bjc.6603554

[15] J. M. Maris, M. D. Hogarty, R. Baqatell and S. L. Cohn, "Neuroblastoma," Lancet, Vol. 369, No. 9579, 2007, pp. 2106-2120. http://dx.doi.org/10.1016/S0140-6736(07)60983-0

[16] K. K. Matthay, J. G. Villablanca, R. C. Seeger, D. O. Stram, R. E. Harris, N. K. Ramsay, P. Swift, H. Shimada, C. T. Black, G. M. Brodeur, R. B. Gerbing and C. P. Reynolds, "Treatment of High-Risk Neuroblastoma with Intensive Chemotherapy, Radiotherapy, Autologous Bone Marrow Transplantation, and 13-cis-Retinoic Acid. Children's Cancer Group," The New England Journal of Medicine, Vol. 341, 1999, pp. 1165-1173. http://dx.doi.org/10.1056/NEJM199910143411601

[17] M. Kaneko, Y. Tsuchida, J. Uchino, T. Takeda, M. Iwafuchi, N. Ohnuma, H. Mugishima, J. Yokoyama, H. Nishihira, K. Nakada, S. Sasaki, T. Sawada, K. Kawa, N. Nagahara, S. Suita and S. Sawaguchi, "Treatment Results of Advanced Neuroblastoma with the First Japanese Study Group Protocol. Study Group of Japan for Treatment of Advanced Neuroblastoma," Journal of Pediatric Hematology/Oncology, Vol. 21, No. 3, 1999, pp. 190-197. http://dx.doi.org/10.1097/00043426-199905000-00006

[18] C Tan, S. M. Sabai, A. S. Tin, T. C. Quah and L. Aung, "Neuroblastoma: Experience from National University Health System, Singapore (1987-2008)," Singapore Medical Journal, Vol. 53, 2012, pp. 19-25.

[19] A. L. Yu, A. L. Gilman, M. F. Ozkaynak, W. B. London, S. G. Kreissman, H. X Chen, M. Smith, B. Anderson, J. G. Villablanca, K. K. Matthay, H. Shimada, S. A. Grupp, R. Seeger, C. P. Reynolds, A. Buxton, R. A. Reisfeld, S. D.
Gillies, S. L. Cohn, J. M. Maris and P. M. Sondel, "Children's Oncology Group. Anti-GD2 Antibody with GMCSF, Interleukin-2, and Isotretinoin for Neuroblastoma," The New England Journal of Medicine, Vol. 363, No. 14, 2010, pp. 1324-1334. http://dx.doi.org/10.1056/NEJMoa0911123

[20] R. C. Seeger, C. P. Reynolds, R. Gallego, D. O. Stram, R. B. Gerbing and K. K. Matthay, "Quantitative Tumor Cell Content of Bone Marrow and Blood as a Predictor of Outcome in Stage IV Neuroblastoma: A Children's Cancer Group Study," Journal of Clinical Oncology, Vol. 18, 2000, pp. 4067-4076.

[21] E. M. Kiely, "Radical Surgery for Abdominal Neuroblastoma," Seminars in Surgical Oncology, Vol. 9, No. 6, 1993, pp. 489-492.

http://dx.doi.org/10.1002/ssu.2980090606

[22] D. von Schweinitz, B. Hero and F. Berthold, "The Impact of Surgical Radicality on Outcome in Childhood Neuroblastoma," European Journal of Pediatric Surgery, Vol. 12, No. 6, 2002, pp. 402-409. http://dx.doi.org/10.1055/s-2002-36952

[23] M. P. La Quaglia, B. H. Kushner, W. Su, G. Heller, K. Kramer, S. Abramson, N. Rosen, S. Wolden and N. K. Cheung, "The Impact of Gross Total Resection on Local Control and Survival in High-Risk Neuroblastoma," Journal of Pediatric Surgery, Vol. 39, No. 3, 2004, pp. 412 417. http://dx.doi.org/10.1016/j.jpedsurg.2003.11.028

[24] A. L. Koivusalo, M. P. Pakarinen, R. J. Rintala and U. M. Saarinen-Pihkala, "Surgical Treatment of Neuroblastoma: Twenty-Three Years of Experience at a Single Institution," Surgery Today, 2013, Epub Ahead of Print. http://dx.doi.org/10.1007/s00595-013-0576-7

[25] A. M. Davidoff, "Neuroblastoma," Seminars in Pediatric Surgery, Vol. 21, No. 1, 2012, pp. 2-14. http://dx.doi.org/10.1053/j.sempedsurg.2011.10.009

[26] S. Mueller and K. K. Matthay, "Neuroblastoma: Biology and Staging," Current Oncology Reports, Vol. 11, No. 6, 2009, pp. 431-438. http://dx.doi.org/10.1007/s11912-009-0059-6 